\title{
Probability estimation in aircraft conflict detection: a simple and computationally effective method with accuracy certificates
}

\author{
Seyedhamed Seyedi Pour, Hadi Nobahari and Maria Prandini
}

\begin{abstract}
In this paper, we address aircraft conflict detection on a mid-term horizon. We adopt a probabilistic approach and provide a simple method to estimate the probability of two aircraft getting closer than some minimum predefined distance, based on a commonly adopted Gaussian model of the deviation from their planned trajectories. A key feature of our method is that the probability estimate is accompanied by a deterministic accuracy certificate. This is achieved by adopting a geometric approach to conflict probability estimation and jointly computing a lower and an upper bound on the conflict probability. These bounds can be inspected and refined if the resulting accuracy of the probability estimate is not adequate. Successive refinements are guaranteed to lead to an estimate with the desired accuracy. Numerical results show the effectiveness of the proposed method.
\end{abstract}

\section{INTRODUCTION}

Demand for air transportation has increased during the recent decades and air traffic is expected to further grow also due to the introduction of unmanned aerial vehicles into the civil airspace. The NextGen, SESAR and CARATS projects, developed in the USA, Europe, and Japan, respectively, aim at increasing airspace capacity through the introduction of new operational concepts where aircraft are not constrained anymore to fly along predefined airways, and through a higher level of automation in the air traffic management system (ATMS), [1], [2]. An essential part of this automation process is the development of effective methods for avoiding that aircrafts get closer than some predefined pairwise distance (conflict situation) or enter forbidden areas of the airspace. Automated conflict detection and resolution (CD\&R) methods are then needed to timely issue an alert and take appropriate resolution actions so that predicted conflicts do not actually occur. The introduction of these support tools will alleviate the tasks of the air traffic controllers that are currently responsible for aircraft safety and have to detect and solve conflicts on a mid-term prediction horizon of about 20 minutes.

This paper aims at improving the existing mid-term conflict detection (CD) approaches by proposing a simple method that guarantees the desired accuracy in conflict prediction while requiring a low computational effort. In the sequel, when referring to $\mathrm{CD}$, we shall mean mid-term $\mathrm{CD}$.

Seyedhamed Seyedi Pour and Hadi Nobahari are with the Department of Aerospace Engineering, Sharif University of Technology, Tehran, Iran, email: seyedipour_seyedhamed@ae.sharif.edu, nobahariesharif.edu

Maria Prandini is with the Dipartimento di Elettronica Informazione e Bioingegneria, Politecnico di Milano, Piazza Leonardo da Vinci 32, 20133 Milano, Italy, email: maria.prandini@polimi.it
Conflicts should be timely predicted with a certain accuracy so as to reduce false alarms and missed detections. One of the difficulties in CD is that the aircraft motion is affected by uncertainty, mainly due to wind, but also to other sources of error related to tracking, navigation, and control.

$\mathrm{CD}$ methods can be classified into nominal, worst-case and probabilistic ones [3], based on the approach adopted for conflict prediction. In nominal CD methods, aircraft are assumed to fly along their nominal trajectories and uncertainty in their future position is neglected, which may cause a large number of missed detections. In worst-case CD methods, all possible future positions are considered equally likely, thus resulting in a large number of false alarms. Finally, probabilistic CD methods lead to a tradeoff between false alarms and missed detections since they do account for uncertainty affecting the aircraft position but weight it according to its likelihood [2]. In this paper, we address CD through a probabilistic approach.

Our goal is to introduce a new method that is computationally efficient and provides an estimate of the instantaneous probability of conflict together with a deterministic bound on its accuracy.

Similarly to most works in the literature on probabilistic CD (see Section II), the uncertainty on the aircraft position is characterized through its deviation from the nominal trajectory and it is Gaussian and independent between aircraft. An over- and under-estimate of the conflict probability is calculated, and the estimate is taken to be their average. The (a-posteriori) accuracy bound is then straightforward to compute and, upon inspection, it can be decreased by refining the under- and over-estimates until the desired accuracy level is obtained.

The rest of the paper is organized as follows. In Section II we provide a short review of methods for probabilistic CD. We then formulate the problem of estimating the instantaneous probability of conflict in Section III. The proposed method is described in Section IV. Section V shows some numerical results where we compare our approach with respect to competing ones in the literature. Some conclusions are drawn in Section VI.

\section{Probabilistic Methods For CD}

Several probabilistic CD methods have been proposed in the literature using analytical [4]-[6], numerical [2], [7][10], and sample-based [11], [12] approaches.

In [4] a simple and fast analytic solution, called geometric method, is proposed. A Gaussian model of the deviation of the aircraft from the nominal trajectory is adopted for the 
level flight case, motivated by a statistical analysis on real air traffic data [13], [14]. Under independence assumption on the two aircraft deviations, the distance between two aircraft is also Gaussian. A conflict occurs if the random variable modeling the aircraft distance enters a circle of radius equal to the safety distance (conflict zone). First, a coordinate transformation is used to convert the two-dimensional (2D) Gaussian probability density function into the product of two 1D Gaussian probability density functions. Then, assuming constant heading and velocity, the (transformed) elliptical conflict zone is extended in one dimension and the integral of the product of the 1D Gaussian densities is computed along the extended conflict zone that models the aircraft encounter. If the heading and velocity are not constant throughout the encounter, then, those at the point of minimum distance are considered. In [5] the approach in [4] is refined by taking the maximum over the prediction horizon of the instantaneous probability of conflict, which is computed by integrating the 2D Gaussian probability density function modeling the aircraft distance over a rectangular over-approximation of the conflict zone. In this way, the two-dimensional integral of the Gaussian density is decoupled into two one-dimensional integrals that are easy to compute. Note that the obtained estimate is an upper bound on the instantaneous probability of conflict, given that an over-approximation of the conflict zone is adopted. The method proposed in [5] for overestimating the instantaneous conflict probability is further improved in [6] by covering the elliptical conflict zone with a finite number of rectangular sets. The estimation error of the geometric method is thus reduced. However, no accuracy bound is provided for the estimation.

In [7], the accumulated risk of a conflict over the time horizon of interest is considered for CD. This accumulated risk is computed by dividing the prediction time horizon $\left[T_{0}, T_{f}\right]$ in time steps $\left[t_{k}, t_{k+1}\right], k=0, \ldots, M-1$, with $T_{0}=t_{0}<t_{1}<\cdots<t_{k}<t_{k+1}<\cdots<t_{M}=T_{f}$, and computing the risk of a conflict over each time step $\left[t_{k}, t_{k+1}\right]$ as the integral over the conflict zone of the probability density function modeling the aircraft distance, conditional to the fact that no conflict occurred in the previous time steps. This involves propagating the conditional probability density function through the dynamics of the two aircraft distance over $\left[t_{k}, t_{k+1}\right]$, and restricting the domain of the propagated density to the conflict zone. Initialization at time $t_{0}=T_{0}$ is provided by a 2D Gaussian probability density function as in [4]. The accumulated risk is the sum of the risks associated with the $M$ time steps. Various approximations are introduced even for a linear model of the dynamics governing the two aircraft distance. In [8], the conflict probability over a time interval for the Gaussian model in [4] is computed based on its flow rate at the boundary of the conflict zone (probability flow). Both the methods in [7] and [8] calculate an upper bound on the conflict probability to reduce the computation time. In [8] the uncertainty affecting the aircraft motion is considered to be Gaussian over the whole reference time horizon, which ease the computations with respect to [7], where only the initial (unconditional) probability density function of the aircraft relative position is assumed to be Gaussian. The accuracy of both methods is degraded when the conflict period is long, such as for parallel scenarios, or when the conflict probability is not negligible, [2], which is the case relevant to conflict detection. In [9], the probability flow is still adopted but decomposed into two contributions, a diffusion and a drift term, in order to ease the calculation of the collision probability between two ships. Still, the same issues of the method in [8] are present. Additionally, to obtain accurate results, a parameter related to the diffusion term has to be tuned. In [10], the cumulative distribution function of the quadratic form of the distance is approximated using a truncated Laurent series expansion, in order to provide an estimate of the instantaneous probability of conflict which is indeed the probability that the (Gaussian) aircraft distance belongs to a circle. The accuracy of the method can be improved by increasing the order of the truncated Laurent series expansion. However, no error bound is provided.

In [11], a randomized algorithm is proposed to estimate the maximum instantaneous conflict probability over a mid-term horizon based on a piecewise linear stochastic differential equation describing the aircraft motion between waypoints. [12] adopts for CD the predicted probabilistic reach sets of the positions occupied by the aircraft, which are computed via a sample-based approach on a 2D nonlinear aircraft motion model subject to a Gaussian random field modeling the wind. Although sample-based algorithms can be applied to general prediction models, they typically require a large number of runs which can be computationally intensive and time demanding for complex dynamics. Also, they can provide an a-priori accuracy bound but only in probability.

\section{PROBLEM Formulation}

Consider two aircraft, say aircraft $\mathrm{S}$ and aircraft $\mathrm{R}$, that are flying at the same constant altitude while following their nominal level flight trajectories. The actual positions $r_{\mathrm{S}}$ and $r_{\mathrm{R}}$ of aircraft $\mathrm{S}$ and $\mathrm{R}$, respectively, may deviate from the nominal ones due to various sources of errors including wind. As a result, $r_{\mathrm{S}}$ and $r_{\mathrm{R}}$ are the sum of the nominal aircraft position and its stochastic deviation:

$$
\begin{aligned}
& r_{\mathrm{S}}=\bar{r}_{\mathrm{S}}+\Delta r_{\mathrm{S}} \\
& r_{\mathrm{R}}=\bar{r}_{\mathrm{R}}+\Delta r_{\mathrm{R}}
\end{aligned}
$$

Now, in order to estimate the instantaneous conflict probability along the reference mid-term horizon, at time $t$ we shall determine the probability that the aircraft distance

$$
d(t)=r_{\mathrm{S}}(t)-r_{\mathrm{R}}(t)
$$

has an absolute value that is smaller than the safety distance. In the following, time dependence is omitted to ease the notation. As suggested, e.g., in [5], we can fictitiously view aircraft $\mathrm{R}$ as the reference aircraft flying with a velocity that is equal to the relative instantaneous nominal velocity of aircraft $S$ with respect to aircraft $R$, and aircraft $S$ as the stochastic aircraft whose distance from aircraft $R$ is given by $d$. Correspondingly, the probability of conflict is 


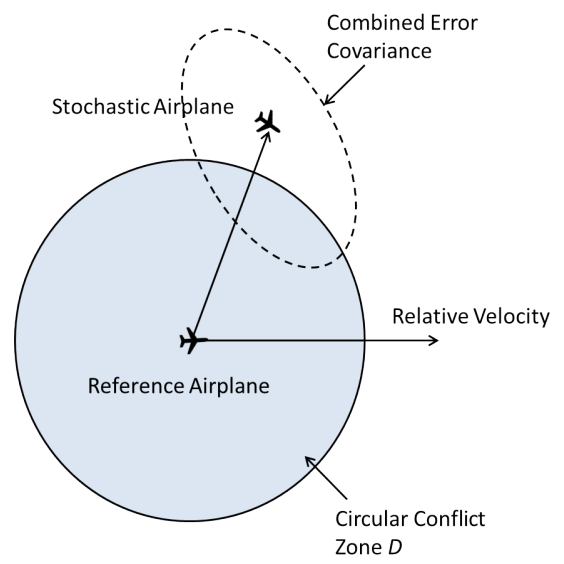

Fig. 1: Conflict zone and combined error covariance [5]

the probability that aircraft $\mathrm{S}$ is within the circular conflict zone $D \subset \Re^{2}$ centered at aircraft $R$ with radius equal to the safety distance. This interpretation is pictorially drawn in Fig. 1 taken from [5]. In formulas, we have that

$$
p_{\mathrm{c}}=\int_{D} f_{d}(s) d s,
$$

where $f_{d}$ denote the probability density function of the $2 \mathrm{D}$ random variable $d$. According to the experimentally assessed model in [4], for mid-term horizon prediction purposes, the tracking errors $\Delta r_{\mathrm{S}}$ and $\Delta r_{\mathrm{R}}$ of the two aircraft can be modeled as two jointly Gaussian random variables with zero mean, covariance matrices $V_{S}$ and $V_{R}$, and cross-covariance matrices $V_{\mathrm{SR}}=E\left[\Delta r_{\mathrm{S}} \Delta r_{\mathrm{R}}^{\top}\right]$ and $V_{\mathrm{RS}}=E\left[\Delta r_{\mathrm{R}} \Delta r_{\mathrm{S}}^{\top}\right]$.

Remark 1: Note that the cross-covariance terms could be used to model the correlation caused by wind [15]. However, it is set to zero in most approaches in the literature. Also, the covariance matrices $V_{S}$ and $V_{R}$ are typically not diagonal, since none of the coordinate axes is generally aligned with the aircraft trajectory and the along-track and cross-track errors are independent but with a different variance.

Correspondingly, $d$ is Gaussian and can be characterized through its mean and covariance matrix:

$$
f_{d}(\cdot)=\mathcal{G}_{2}(\cdot ; \mu, V),
$$

where $\mu=\bar{r}_{\mathrm{S}}-\bar{r}_{\mathrm{R}}$ can be computed based on the nominal trajectories and $V=V_{\mathrm{S}}+V_{\mathrm{R}}-V_{\mathrm{SR}}-V_{\mathrm{RS}}$. By plugging in this expression for $f_{d}$ in (2) one finally obtains:

$$
p_{\mathrm{c}}=\int_{D} \mathcal{G}_{2}(s ; \mu, V) d s .
$$

The issue of efficiently computing (3) guaranteeing a high accuracy in a limited computation time is addressed in the next section.

\section{PRoposed METHOD}

The conflict probability in (3) cannot be calculated analytically because it involves integrating a 2D Gaussian density function. However, if it can be reduced to computing integrals of 1D Gaussian densities, then, a high quality approximation of each single 1D integral can be obtained so that the resulting method is classified as analytical (see Section II). This is the idea exploited in the geometric methods in [4]-[6] and also in the one proposed here, which is a simple extension of the method proposed in [6]. Despite its simplicity, this extension has the twofold positive effect of improving the quality of the estimate of the conflict probability and also providing a deterministic bound on its accuracy. This is indeed a key distinguishing feature of our method and allows the user to decide if possibly refining the estimate upon inspection of its value and accuracy. This is useful, for example, in the case of a not much accurate estimate that is close to the threshold value set for issuing an alert in a CD system.

In order to compute our estimate and accuracy bound, we first apply a coordinate transformation to reduce the covariance matrix $V$ in (3) to an identity matrix so that the two component of the distance in the new reference frame are independent with unitary variance and keep independent and with unitary variance for any rotation of the coordinate system. This transformation rests on the Cholesky factorization of the positive definite covariance matrix $V$ :

$$
V=L^{\top} L
$$

where $L$ is upper triangular with positive elements on the diagonal. If we set the new coordinate variables as

$$
\left[\begin{array}{l}
\xi \\
\eta
\end{array}\right]=L\left[\begin{array}{l}
x \\
y
\end{array}\right]
$$

where $x$ and $y$ are the original coordinates, then, in the new coordinate system $d$ is still Gaussian but with the following mean and variance:

$$
\tilde{\mu}=L \mu=\left[\begin{array}{c}
\tilde{\mu}_{\xi} \\
\tilde{\mu}_{\eta}
\end{array}\right] \text { and } \tilde{V}=I
$$

so that

$$
\mathcal{G}((\xi, \eta) ; \tilde{\mu}, \tilde{V})=\mathcal{G}\left(\xi ; \tilde{\mu}_{\xi}, 1\right) \cdot \mathcal{G}\left(\eta ; \tilde{\mu}_{\eta}, 1\right),(\xi, \eta) \in \Re^{2} .
$$

Correspondingly, the circular conflict zone $D$ is reshaped into an ellipse $\tilde{D}$ still centered at the origin as it was $D$. Without loss of generality, it can be assumed that the major axis of the ellipse $\tilde{D}$ is parallel to the vertical axis $\eta$. As a matter of fact, if this were not the case, a suitable rotation (see e.g. [16, p. 979]) can be applied to the coordinate system so as to make the major axis of $\tilde{D}$ aligned with the vertical axis while preserving the property that the variance matrix of $d$ is the identity matrix.

Finally, equation (3) can be rewritten as

$$
p_{\mathrm{c}}=\int_{\tilde{D}} \mathcal{G}\left(\xi ; \tilde{\mu}_{\xi}, 1\right) \cdot \mathcal{G}\left(\eta ; \tilde{\mu}_{\eta}, 1\right) d \xi d \eta .
$$

If the ellipse $\tilde{D}$ is approximated by the union of rectangular sets with sides aligned with the coordinate axes, then, the integral in (5) can be approximated by the sum of the integrals over the rectangular sets, which in turn reduced to the product of two integral involving $\mathcal{G}\left(\cdot ; \tilde{\mu}_{\xi}, 1\right)$ and $\mathcal{G}\left(\cdot ; \tilde{\mu}_{\eta}, 1\right)$, separately. Both an upper and a lower bound 


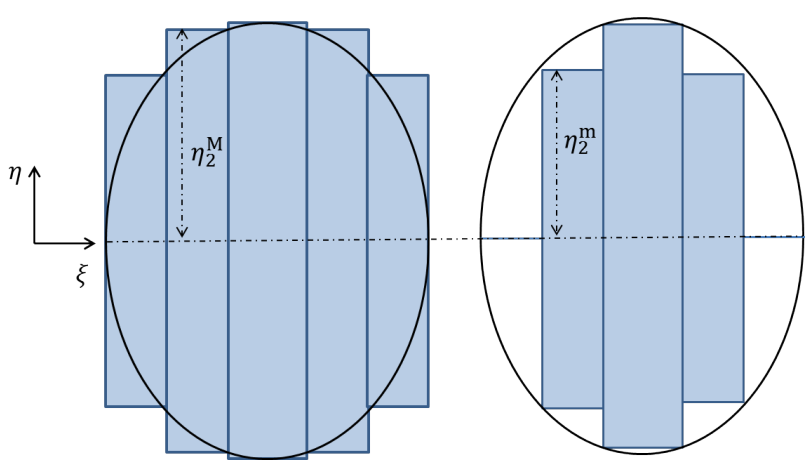

Fig. 2: Outer and inner approximation of the conflict zone through $N=5$ rectangular sets.

on the conflict probability can be determined by adopting an inner and an outer approximation of the ellipse through rectangular sets. An example is shown in Fig. 2.

Only upper bounds on the conflict probability have been introduced in the current relevant literature. Here, we determine both an upper bound $p_{\mathrm{c}}^{\mathrm{UB}}$ and a lower bound $p_{\mathrm{c}}^{\mathrm{LB}}$ on the conflict probability, and define its estimate as their average

$$
\hat{p}_{\mathrm{c}}=\frac{p_{\mathrm{c}}^{\mathrm{UB}}+p_{\mathrm{c}}^{\mathrm{LB}}}{2} .
$$

The quality of the estimate $\hat{p}_{\mathrm{c}}$ in (6) can be quantified via an accuracy parameter $\epsilon$ such that $p_{\mathrm{c}} \in\left(\hat{p}_{\mathrm{c}}-\epsilon, \hat{p}_{\mathrm{c}}+\epsilon\right)$, where

$$
\epsilon=\frac{p_{\mathrm{c}}^{\mathrm{UB}}-p_{\mathrm{c}}^{\mathrm{LB}}}{2} .
$$

We shall next explain in some detail how we compute $p_{\mathrm{c}}^{\mathrm{UB}}$ and $p_{\mathrm{c}}^{\mathrm{LB}}$. Note that in [6], only the upper bound $p_{\mathrm{c}}^{\mathrm{UB}}$ is computed and the conflict probability estimate is set equal to such an upper bound without providing any accuracy. This prevents the possibility of implementing a guided refinement so as to achieve some desired quality of the estimate.

\section{A. Upper and lower bound computation}

Let us partition the minor axis of the ellipsoidal conflict zone centered in the origin of the $(\xi, \eta)$ plane with the major axis aligned with the vertical $\eta$ axis into $N$ intervals $\left[\xi_{i}^{-}, \xi_{i}^{+}\right)$, $i=1, \ldots, N$, of the same length as shown in both plots of Fig. 2. Consider now the points $(\xi, \eta)$ of the ellipse with $\xi=\xi_{i}^{-}$and $\xi=\xi_{i}^{+}$that belong to the positive $\eta$ half plane. Let $\eta_{i}^{\mathrm{M}}$ and $\eta_{i}^{\mathrm{m}}$ be the largest and smallest values of the resulting $\eta$ coordinates (these two quantities are shown for the second rectangular set in Fig. 2). Then, we can compute an upper bound on the conflict probability as

$$
p_{\mathrm{c}}^{\mathrm{UB}}=\sum_{i=1}^{\mathrm{N}} p_{\mathrm{c}, i}^{\mathrm{UB}},
$$

where

$$
p_{\mathrm{c}, i}^{\mathrm{UB}}=\left(\Psi_{\tilde{\mu}_{\eta}}\left(\eta_{i}^{\mathrm{M}}\right)-\Psi_{\tilde{\mu}_{\eta}}\left(-\eta_{i}^{\mathrm{M}}\right)\right) \cdot\left(\Psi_{\tilde{\mu}_{\xi}}\left(\xi_{i}^{+}\right)-\Psi_{\tilde{\mu}_{\xi}}\left(\xi_{i}^{-}\right)\right)
$$

with $\Psi_{\alpha}(x):=\int_{-\infty}^{x} \mathcal{G}(v ; \alpha, 1) d v=\int_{-\infty}^{x-\alpha} \mathcal{G}(v ; 0,1) d v$.
Analogously, we can compute a lower bound on the probability of conflict as

$$
p_{\mathrm{c}}^{\mathrm{LB}}=\sum_{i=1}^{\mathrm{N}} p_{\mathrm{c}, i}^{\mathrm{LB}},
$$

where

$p_{\mathrm{c}, i}^{\mathrm{LB}}=\left(\Psi_{\tilde{\mu}_{\eta}}\left(\eta_{i}^{\mathrm{m}}\right)-\Psi_{\tilde{\mu}_{\eta}}\left(-\eta_{i}^{\mathrm{m}}\right)\right) \cdot\left(\Psi_{\tilde{\mu}_{\xi}}\left(\xi_{i}^{+}\right)-\Psi_{\tilde{\mu}_{\xi}}\left(\xi_{i}^{-}\right)\right)$.

If we grow $N$, then, the accuracy (7) of the conflict probability estimate in (6) improves.

\section{Numerical Results}

In this section, we compare the proposed approach with the geometric approaches in [4], [5], and [6] through two numerical examples. Results are reported in Sections V$\mathrm{A}$ and V-B. In both examples, we consider an encounter where two aircrafts are flying at the same constant altitude, each one following a nominal trajectory traveled at constant velocity. This is the framework where the method in [4] is more accurate. In order to set a baseline for all methods, the conflict probability is also evaluated through a highly accurate but time consuming method, called the "exact method", resorting to the numerical integration of the probability density function of the distance (1) using a fine uniform mesh of the conflict zone with equilateral triangles. The mesh is generated by using the DistMesh toolbox [17] with the initial edge length set to $0.0625 \mathrm{nmi}$ (nautical miles). This parameter is the initial distance between the corner points of the triangles. According to the standards for enroute flight, the safety distance is set equal to $5 \mathrm{nmi}$. Computations are performed with MATLAB on a Dell 1320 Notebook Computer.

Note that the accuracy of the conflict probability estimate can be improved in our method and the one proposed in [6] by increasing the number $N$ of rectangular sets approximating the conflict zone, whereas the accuracy of the methods in [4] and [5] is fixed for a specified conflict geometry and cannot be improved. Furthermore, in our method we can decide if further increasing $N$ and improving the estimate by inspecting the obtained accuracy $\epsilon$.

\section{A. Numerical example with fixed relative heading}

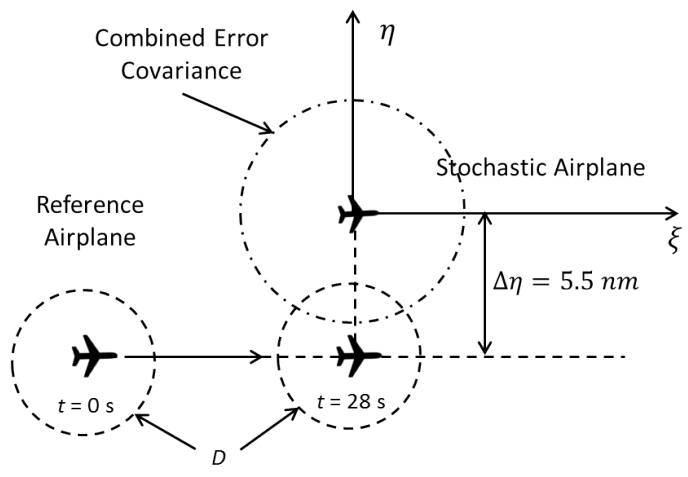

Fig. 3: Encounter geometry [5] - example of Section V-A. 

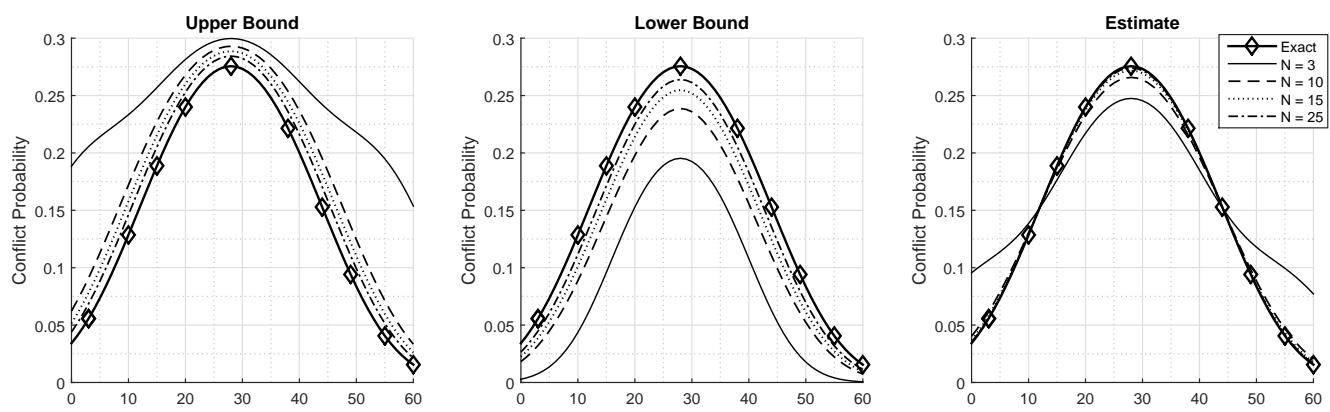

Fig. 4: Lower bound, upper bound, and estimate of the conflict probability obtained with the method proposed in this paper for a number of rectangular sets $N \in\{3,10,15,25\}$ - example of Section V-A.

We consider the 2D encounter in Fig. 3 taken from [5], where the stochastic aircraft is at the origin of the coordinate frame and the reference one flies with a constant horizontal velocity at a distance of $5.5 \mathrm{nmi}$ along the vertical axis. The combined error covariance $V$ is assumed to be constant during the encounter and equal to the identity matrix when measured in $\mathrm{nmi}^{2}$.

In Fig. 4, we plot lower bound, upper bound and estimate of the conflict probability obtained with the method proposed in this paper, for a growing number $N$ of rectangular sets.
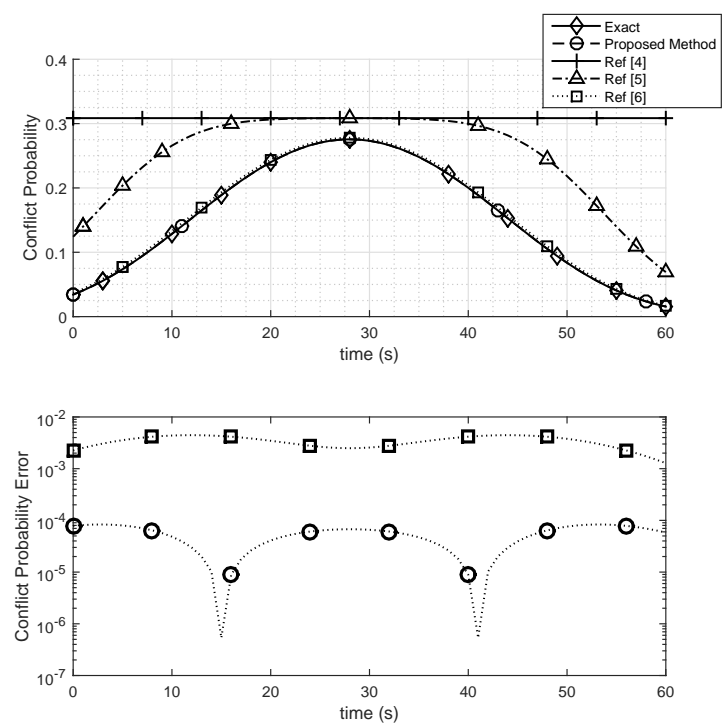

Fig. 5: Top plot: Conflict probability estimate computed with our method, those in [4], [5], [6], and the exact method. Bottom plot: error of the estimate obtained with our method and that in [6] using $N=100$ rectangular sets - example of Section V-A.

The results of conflict probability estimation obtained with our method, the methods in [4], [5], and [6], and the exact method are reported in the top plot of Fig. 5, using $N=100$ for both our method and that in [6]. The results show that the method proposed in [4] as well as that in [5] largely overapproximate the probability of conflict and has, hence, low accuracy. On the contrary, the method proposed here and the method proposed in [6] have both comparable accuracy and provide an estimate that is very close to that obtained with the the exact method since $N$ is large $(N=100)$. The estimation error is shown in the bottom plot of Fig. 5.

\section{B. Numerical example with different relative headings}

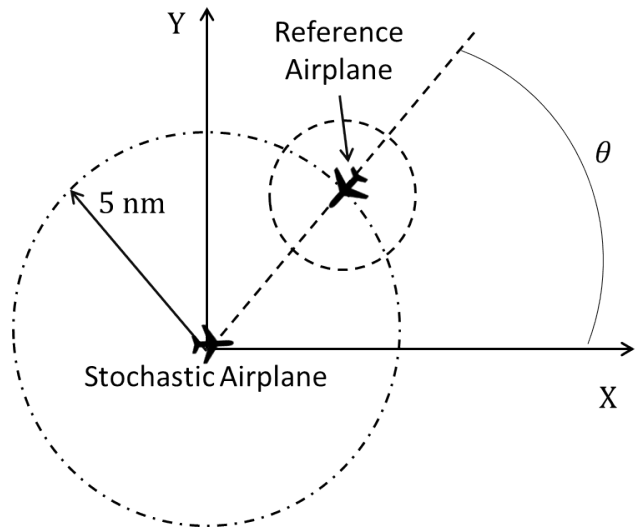

Fig. 6: Encounter geometry - example of Section V-B.

We consider the 2D scenario in Fig. 6, where the stochastic aircraft is at the origin whereas the reference aircraft is on the circle of radius $5 \mathrm{nmi}$ at an angular position $\theta$ with a velocity that is pointing towards the stochastic aircraft.

The conflict probability at time 0 is calculated for $\theta$ ranging from 0 to 180 degree with a step of 10 degrees, assuming that the combined error covariance $V$ is equal to the identity matrix.

Fig. 7 shows the error in the conflict probability estimation and the computation time as a function of the number of the rectangular sets $(N)$ used in our method and in the method proposed in [6]. For each $N$, average values with respect to all $\theta$ 's are reported. As for the computation time, it is determined per each $\theta$ value as the mean of the computation times obtained by repeating calculations 1000 times.

In both methods, the accuracy is improved at the expense of the computation time. However, the error of the method proposed here decays much faster.

The computation time of the exact method, the method in [6], and the one proposed in this paper are reported in Table I, together with the conflict probability error. This error 

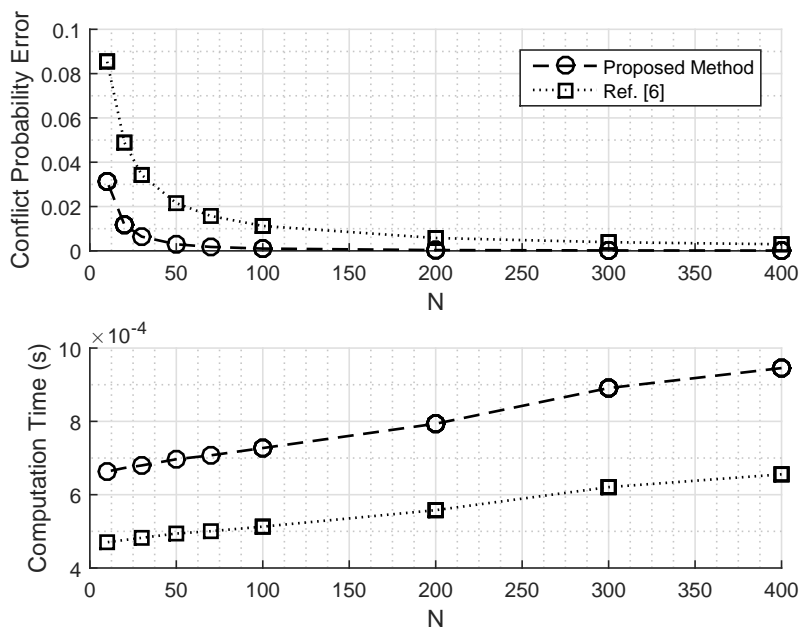

Fig. 7: Conflict probability estimation error and computation time as a function of the number $N$ of rectangular sets for the proposed method and one described in [6] - example of Section V-B.

is determined by considering the conflict probability value calculated by the exact method with the finest mesh (initial edge length set equal to $0.0625 \mathrm{nmi}$ ) as if it were the true conflict probability. In order to show the trade-off between the computation time and the conflict probability error of the exact method, two rougher triangular meshes with initial edge lengths set equal to 2 and 10 times of the default value $(0.0625 \mathrm{nmi})$ are considered. The results indicate that the exact method outperform both the approximation methods in [6] and in this paper in terms of accuracy if a small-enough mesh is used. However, this comes at the expense of a much larger computation time.

TABLE I: Conflict probability error and computation time of the exact method, the method described in [6], and the proposed method- example of Section V-B

\begin{tabular}{ccccc} 
Method & $\begin{array}{c}\text { Initial Edge } \\
\text { Length (nmi) }\end{array}$ & $N$ & $\begin{array}{c}\text { Conflict } \\
\text { Probability Error }\end{array}$ & $\begin{array}{c}\text { Computation } \\
\text { Time }(\mathrm{s})\end{array}$ \\
\hline exact & $2 \times 0.0625$ & - & $4.0 \mathrm{e}-6$ & 2.3 \\
exact & $10 \times 0.0625$ & - & $7.0 \mathrm{e}-3$ & 0.07 \\
[6] & - & 200 & $5.8 \mathrm{e}-3$ & $5.6 \mathrm{e}-4$ \\
[6] & - & 400 & $3.0 \mathrm{e}-3$ & $6.6 \mathrm{e}-4$ \\
proposed & - & 200 & $3.4 \mathrm{e}-4$ & $7.6 \mathrm{e}-4$ \\
proposed & - & 400 & $1.2 \mathrm{e}-4$ & $9.5 \mathrm{e}-4$
\end{tabular}

\section{CONCLUSIONS}

In this paper, we addressed mid-term probabilistic $\mathrm{CD}$ for two aircraft encounters in the level flight case. We devel- oped a new method to compute an estimate of the conflict probability. The distinguishing feature of our method is that it provides a bound on the accuracy of the estimate, which can be used to decide if some refinement of the estimate is needed. We presented two numerical examples and showed that the method is promising and better performing with respect to alternative methods available in the literature. Further work is needed to extend it to the 3D airspace where aircraft are not necessarily flying at the same constant altitude.

\section{REFERENCES}

[1] N. Yokoyama, "Decentralized conflict detection and resolution using intent-based probabilistic trajectory prediction," in 2018 AIAA Guidance, Navigation, and Control Conference, 2018, p. 1857.

[2] L. J. Pienaar and T. Jones, "The application of probability flow for conflict detection near airports," in AIAA Guidance, Navigation, and Control Conference, 2015, p. 1325.

[3] J. K. Kuchar and L. C. Yang, "A review of conflict detection and resolution modeling methods," IEEE Transactions on intelligent transportation systems, vol. 1, no. 4, pp. 179-189, 2000.

[4] R. A. Paielli and H. Erzberger, "Conflict probability estimation for free flight," Journal of Guidance, Control, and Dynamics, vol. 20, no. 3, pp. 588-596, 1997.

[5] I. Hwang and C. E. Seah, "Intent-based probabilistic conflict detection for the next generation air transportation system," Proceedings of the IEEE, vol. 96, no. 12, pp. 2040-2059, 2008.

[6] K.-Y. Baek and H.-C. Bang, "ADS-B based trajectory prediction and conflict detection for air traffic management," International Journal of Aeronautical and Space Sciences, vol. 13, no. 3, pp. 377-385, 2012.

[7] T. Jones, "Tractable conflict risk accumulation in quadratic space for autonomous vehicles," Journal of guidance, control, and dynamics, vol. 29, no. 1, pp. 39-48, 2006.

[8] C. E. Van Daalen and T. Jones, "Fast conflict detection using probability flow," Automatica, vol. 45, no. 8, pp. 1903-1909, 2009.

[9] J. Park and J. Kim, "Predictive evaluation of ship collision risk using the concept of probability flow," IEEE Journal of Oceanic Engineering, vol. 42, no. 4, pp. 836-845, 2017.

[10] W. Liu and I. Hwang, "Probabilistic trajectory prediction and conflict detection for air traffic control," Journal of Guidance, Control, and Dynamics, vol. 34, no. 6, pp. 1779-1789, 2011.

[11] M. Prandini, J. Hu, J. Lygeros, and S. Sastry, "A probabilistic approach to aircraft conflict detection," IEEE Transactions on intelligent transportation systems, vol. 1, no. 4, pp. 199-220, 2000.

[12] Y. Yang, J. Zhang, K.-Q. Cai, and M. Prandini, "Multi-aircraft conflict detection and resolution based on probabilistic reach sets," IEEE Transactions on Control Systems Technology, vol. 25, no. 1, pp. 309316, 2017.

[13] M. Ballin and H. Erzberger, "An analysis of landing rates and separations at Dallas/Ft. Worth Airport," NASA Technical Memorandum, TM 110397, Tech. Rep., July 1996.

[14] R. Paielli, "Empirical test of conflict probability estimation," in 2st USA/Europe Air Traffic Manegement R \& D Seminar, 1998.

[15] I. Lymperopoulos, "Sequential monte carlo methods in air traffic management," Ph.D. dissertation, ETH Zurich, 2010.

[16] C. Y. Young, Precalculus, 2nd ed. John Wiley \& Sons, 2013.

[17] P.-O. Persson and G. Strang, "A simple mesh generator in matlab," SIAM review, vol. 46, no. 2, pp. 329-345, 2004. 